\title{
The Effect of Neoadjuvant Therapies for Patients with Locally Advanced Gastric Cancer: A Propensity Score Matching Study
}

Tongbo Wang ${ }^{1^{*}}$, Yingtai Chen ${ }^{1 *}$, Lulu Zhao ${ }^{1^{*}}$, Hong Zhou ${ }^{1}$, Chaorui $\mathrm{Wu}^{1}{ }^{1}$, Xiaojie Zhang ${ }^{1}$, Aiping Zhou ${ }^{2^{\bowtie}}$, Jing $\mathrm{Jin}^{3 凶}$ and Dongbing Zhao ${ }^{1 凶}$

1. Department of Pancreatic and Gastric Surgical Oncology, National Cancer Center/ National Clinical Research for Cancer/Cancer Hospital, Chinese Academy of Medical Sciences and Peking Union Medical College, Beijing 100021, China.

2. Department of Medical Oncology, National Cancer Center/National Clinical Research for Cancer/Cancer Hospital, Chinese Academy of Medical Sciences and Peking Union Medical College, Beijing 100021, China.

3. Department of Radiation Oncology, National Cancer Center/ National Clinical Research for Cancer/Cancer Hospital, Chinese Academy of Medical Sciences and Peking Union Medical College, Beijing 100021, China.

*These authors contributed equally to this work.

$\square$ Corresponding authors: Aiping Zhou, M.D., Ph.D., Department of Medical Oncology, National Cancer Center/National Clinical Research for Cancer/Cancer Hospital, Chinese Academy of Medical Sciences and Peking Union Medical College, Beijing 100021, China. E-mail: zhouap1825@126.com, Tel.: +86-13691161998; Jing jin, M.D., Ph.D., Department of Radiation Oncology, National Cancer Center/ National Clinical Research for Cancer/Cancer Hospital, Chinese Academy of Medical Sciences and Peking Union Medical College, Beijing 100021, China. E-mail: jingjin1025@163.com; Tel.: +86-13601365130 \& Dongbing Zhao, M.D., Ph.D., Department of Pancreatic and Gastric Surgical Oncology, National Cancer Center/ National Clinical Research for Cancer/Cancer Hospital, Chinese Academy of Medical Sciences and Peking Union Medical College, Beijing 100021, China. E-mail: dbzhao@cicams.ac.cn; Tel.: +86-13901331816.

(c) The author(s). This is an open access article distributed under the terms of the Creative Commons Attribution License (https://creativecommons.org/licenses/by/4.0/). See http://ivyspring.com/terms for full terms and conditions.

Received: 2020.04.09; Accepted: 2020.10.30; Published: 2021.01.01

\begin{abstract}
Background: The aim of this study was to evaluate the effect of neoadjuvant therapies (NAT) on patients with locally advanced gastric cancer (LAGC).

Methods: This study retrospectively analyzed LAGC patients treated at the China National Cancer Center between October 2006 and December 2018. All patients included were divided into two groups, NAT followed by surgery (NAT-Surgery) and adjuvant chemotherapy following surgery (Surgery-ACT). Subgroup analysis compared between patients underwent either neoadjuvant chemotherapy (nCT) or neoadjuvant chemoradiation ( $\mathrm{nCRT}$ ) was conducted. Propensity score matching (PSM) was implemented to reduce selection bias.

Results: In total, 2779 patients were included in this study (494 of NAT-Surgery group and 2285 of Surgery-ACT group). After PSM, the patients in NAT-Surgery group had a significantly longer overall survival (OS) than patients in Surgery-ACT group $(P<0.00 \mathrm{I})$. Subgroup analysis revealed that grade 3 or 4 adverse events were more frequently observed in nCRT group during neoadjuvant treatment $(52.0 \%$ in $n C R T$ group vs. $34.0 \%$ in $n C T$ group, $P=0.010$ ). Pathological complete response ( $P C R$ ) being achieved in $17.0 \%$ after $\mathrm{nCRT}$ versus $4.0 \%$ after $\mathrm{nCT}(P<0.00 \mathrm{I})$. Patients of the $\mathrm{nCRT}$ group obtained better disease-free survival (DFS, $P=0.024$ ) and local-recurrence-free survival (LRFS, $P=0.014$ ) than patients in $\mathrm{nCT}$ group, while there was no significant difference in OS between the two groups.

Conclusions: In conclusion, NAT improved survival outcomes among LAGC patients over surgery followed by adjuvant chemotherapy. In comparison with $\mathrm{nCT}$, $\mathrm{nCRT}$ resulted in higher $\mathrm{PCR}$ rate, better DFS and LRFS, without significantly affecting OS.
\end{abstract}

Key words: locally advanced gastric cancer, neoadjuvant therapies, adjuvant chemotherapy, neoadjuvant chemotherapy, neoadjuvant chemoradiation 


\section{Introduction}

Gastric cancer is the third leading cause of cancer-related mortality worldwide [1]. At the time of diagnosis, more than $50 \%$ of patients with gastric cancer have locally advanced disease [2]. Despite radical resection, most patients with locally advanced gastric cancer (LAGC) develop recurrence and the 5 -year survival rates remain below 50\% [3, 4]. Thus, the need to find a way to improve survival in locally advanced gastric cancer (LAGC) patients has led to the creation of the so-called "multimodality" strategy that included pre- and post-operative approaches.

Neoadjuvant and adjuvant therapies are generally accepted to improve disease-free survival (DFS) and overall survival (OS) for LAGC patients who have undergone curative resection, however, little consensus exists on the optimum strategy. Perioperative chemotherapy additional to R0 resection is the most popular strategy in Europe, whereas in the USA it is postoperative chemoradiotherapy, and in Asia it is postoperative chemotherapy [5]. These recommendations are based on US 0116 trial [6], MAGIC trail [7] and FNCLCC/FFCD ACCORD trial [3], which showed survival benefits with postoperative chemoradiotherapy and perioperative chemotherapy, respectively, compared with surgery alone. In Asia, the recommendation for postoperative chemotherapy is based on ACTS-GC trial [8] and CLASSIC trial [9], which showed a survival benefit with adjuvant chemotherapy after D2 gastrectomy compared with surgery alone. However, there are limited studies that compare the outcomes between these treatment options despite there are some ongoing trials such as NCT01515748 and phase III TOPGEAR trail. In addition, adding radiation therapy to neoadjuvant therapy for LAGC is still considered investigational.

As such, we conducted this retrospective study with the primary aim of comparing the long-term survival between patients of LAGC treated with and without neoadjuvant therapies (NAT). The secondary aim of this study was to assess the short-term and long-term outcomes regarding the two NAT concepts, neoadjuvant chemotherapy ( $\mathrm{nCT}$ ) and neoadjuvant chemoradiation (nCRT). The evaluation was performed by propensity score matching (PSM).

\section{Methods}

\section{Patients}

From October 2006 to December 2018, patients with clinical stage II-III gastric cancer treated with curative gastrectomy following neoadjuvant therapy (NAT-Surgery) or curative gastrectomy followed by adjuvant chemotherapy (Surgery-ACT) at China National Cancer Center were retrospectively reviewed. Inclusion criteria were as follows: presence of locally advanced (cT2-4a and/or $\mathrm{N}+$ ) gastric adenocarcinoma according to the TNM stage system (American Joint Committee on Cancer, 8th edition); absence of distant metastases confirmed by clinical examination and imaging techniques; no other primary malignancy in the previous 5 years; an Eastern Cooperative Oncology Group performance status (PS) of 0-1. All study procedures were approved by the Institutional Review Board at the China National Cancer Center.

Pretreatment patient evaluation included clinical examination, blood tests, upper gastrointestinal endoscopy \pm endoscopic ultrasound (EUS), enhanced chest-abdominal-pelvic enhanced computed tomography-scan (CT-scan) to determine the extent of the disease.

\section{Neoadjuvant therapies}

Neoadjuvant therapies included of neoadjuvant chemotherapy (nCT group) and neoadjuvant chemoradiation (nCRT group). All the patients were evaluated by a multidisciplinary team including gastrointestinal surgeons, medical oncologists, radiation oncologists and radiologists. Based on the patient's age, comorbidities, clinical TNM stage, and a pretreatment evaluation consisted of physical examination, complete blood count, hepatic function, serum tumor marker assessment and electrocardiogram, the most suitable therapeutic strategy was recommended.

\section{Neoadjuvant chemotherapy}

All patients from nCT group received 4-6 cycles of chemotherapy at the Department of Medical Oncology. Chemotherapy regimens consisted of SOX (S-1/oxaliplatin), FOLFOX (oxaliplatin/leucovorin/ fluorouracil) and taxane-based therapy of FOLT (fluorouracil/leucovorin/oxaliplatin/docetaxel) and DOS (S-1/oxaliplatin/docetaxel).

\section{Neoadjuvant chemoradiation}

For patients from nCRT group, neoadjuvant therapeutic settings included induction chemotherapy followed by concurrent chemoradiation (induction CT+CCRT) and CCRT alone. Induction CT was based on the regimen of SOX as described above for 2-4 cycles. For CCRT planning, patients received two irradiation dose levels of planning target volume (PTV) and PTV boost of 40.04 and 45.1 Gy 22 fractions plus $\mathrm{S}-1\left(80 \mathrm{mg} / \mathrm{m}^{2} / \mathrm{d}\right)$ administrated on radiotherapy days. Radiotherapy was delivered to the entire stomach and draining regional lymph nodes using 
three-dimensional (3D) conformal techniques, intensity-modulated radiotherapy (IMRT).

CT-scan and / or positron emission tomography (PET) scan was performed during the therapy to monitor the clinical response. The tumor response was classified according to the Response Evaluation Criteria in Solid Tumors (RECIST) [10] and the adverse events of chemotherapy were assessed according to the National Cancer Institute-Common Terminology Criteria for Adverse Events (NCI-CTCAE version 4.0).

\section{Surgery}

For all patients included in the present study, the type of gastrectomy, totally or sub-totally, was determined by the size and location of the primary tumor. For patients underwent NAT, Surgery was scheduled 4-6 weeks after the completion of neoadjuvant treatment. D2 lymphadenectomy with spleen preservation was recommended for all patients. Postoperative complications were defined according to the Clavien-Dindo classification [11].

\section{Pathological assessment}

All resected specimens were reviewed by two pathologists to evaluate the pathological response to neoadjuvant treatment and the extent of the residual disease. The pathological tumor staging was determined according to the American Joint Committee on Cancer (AJCC) TNM Staging Classification for Carcinoma of the Stomach, $8^{\text {th }}$ edition. A complete pathological response ( $\mathrm{pCR}$ ) was considered when no evidence of residual tumor was found in the surgical specimen (ypT0N0). The histological grade of tumor regression was classified based on the Mandard tumor regression grade (TRG) [12].

\section{Postoperative management and Follow-up}

Postoperative adjuvant chemotherapy was recommended for all patients included in the present study. The regimens of postoperative adjuvant chemotherapy were consistent with the preoperative part of treatment. However, some patients declined postoperative chemotherapy due to various reasons. All the patients were followed up every 3 months for the first 3 years and every 6 months afterwards until 5 years post-surgery. The follow-up content included physical examination, complete blood count, hepatic function, serum tumor marker assessment, and CT-scan as well as annual gastrointestinal endoscopy.

\section{Propensity score matching}

In an attempt of reducing selection bias and confounding, two sets of cohorts (NAT-Surgery vs. Surgery-ACT, and nCT vs. nCRT) were created using PSM methods at a ratio of 1:1. Propensity scores were estimated using a logistic regression model and including the following characters: age, gender, year of diagnosis, tumor location, grade, clinical T-stage, clinical N-stage, clinical TNM stage (AJCC, $8^{\text {th }}$ edition). PSM was performed using SPSS v25 (IBM Corp., Chicago, IL, USA) with a caliper size of 0.02 .

\section{Statistics}

Categorical variables were expressed as numbers and percentages, and continuous variables were expressed as the mean \pm standard deviation (SD). The Kaplan-Meier method was used to analyze the survival data, the log-rank test to compare the survival rates. The OS was calculated from the date of surgery to the date of death or last contact. The DFS was defined as the time from surgery to the date of recurrence or metastasis. The LRFS was defined as the time from surgery to the date of local recurrence. The statistical analysis was performed with SPSS v25 (IBM Corp., Chicago, IL, USA) and Graphpad Prism 7 (GraphPad Software, San Diego, California, USA). A P $<0.05$ was considered to be statistically significant.

\section{Results}

\section{Patients' characteristics}

A total of 2779 patients were identified and included in this study (Figure 1). Patients were categorized into two groups according to the treatment strategies, 494 of NAT-Surgery group and 2285 of Surgery-ACT group. Patients' characteristics and tumor baseline parameters were shown in Supplement Table 1. Tumor location, grade, clinical $\mathrm{T}$ stage, clinical $\mathrm{N}$ stage and clinical TNM stage (AJCC $8^{\text {th }}$ edition) were significantly different between the two groups. We conducted two sets of propensity score matching (NAT-Surgery vs. Surgery-ACT and nCRT vs. nCT) to balance the baseline differences between groups for outcome comparison (Table 1).

\section{Long-term survival comparison between NAT- Surgery and Surgery-ACT}

After PSM, 389 pairs of patients were successfully matched (Table 1). Over a median follow-up of 47.0 months (range, 0.6-137.8 months), the median OS for patients in NAT-Surgery group was 52.0 months and for Surgery-ACT group was 26.4 months. The patients in NAT-Surgery group had a significant longer OS than patients in Surgery-ACT group (Hazard ratio [HR], 0.45; 95\% Confidential interval [CI], 0.35-0.58; $P<0.001$, Figure 2).

\section{Neoadjuvant therapies induced toxicity effects}

To further assess the outcomes of the two neoadjuvant treatments, we conducted PSM between nCRT group and nCT group and 100 pairs of patients 
were matched (Table 1). All severe adverse events that occurred during neoadjuvant treatment were summarized in Table 2 . In the propensity score matched analysis, $29.0 \%$ of the patients in nCRT group and $14.0 \%$ in $\mathrm{nCT}$ group had grade 3 to 4 neutropaenia and thrombocytopaenia during neoadjuvant treatment $(P=0.010)$. There was no difference between two groups in the frequency of other adverse events regarding nausea/vomiting, gastrointestinal symptoms, anemia, dyspepsia, infection, gastrointestinal bleeding, hand-foot syndrome and gastritis/esophagitis. In total, grade 3 or 4 adverse events were more frequently observed in nCRT group during neoadjuvant treatment $52.0 \%$ in nCRT group vs. $34.0 \%$ in nCT group, $P=0.010$ ).

\section{Surgical outcomes comparison between nCRT and $\mathrm{nCT}$}

Surgical outcomes and postoperative complications were listed in Table 3. The mean blood loss of nCRT group was $108 \mathrm{~mL}$ less than that of nCT group (170 vs. $278 \mathrm{~mL}, P<0.001)$. Moreover, the intraoperative blood transfusion rate of nCRT group was lower than that of nCT group (15.0 vs. $29.0 \%$, $P=0.017)$ and the mean postoperative hospital stay was also shorter in nCRT group (11 vs. 12 days, $P=0.005)$. However, there was no difference in mean surgical time between these two groups. The total medical expenditure was comparable between the two groups $(P=0.873)$.

Postoperative mortalities and morbidities were presented in Table 3. One patient in nCT group died within 30 days after surgical resection. Totally, the rate of postoperative complications was $9.0 \%$ and $16.0 \%$ for nCRT and nCT group $(P=0.134)$, respectively.

\section{2 patients were retrospective reviewed (2006-2018) \\ Histologically confirmed gastric adenocarcinoma \\ Treated with NAT-Surgery or Surgery-ACT}

633 Excluded

309 incomplete clinical data registered

53 other primary malignancy in the previous 5 years

156 non-curable surgical resection

115 M1 disease

2779 patients were included

CT2-4 and/or $\mathrm{N}+$; MO disease

no other primary malignancy in the previous 5 years

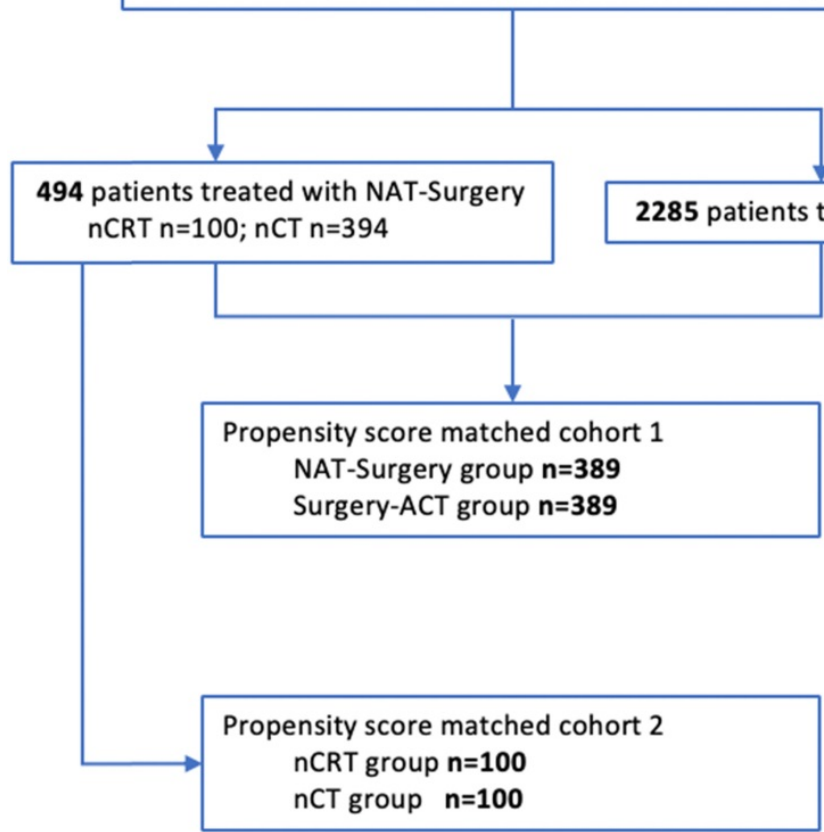

Figure I. Patient Flowchart. 
Table I. Baseline characteristics of the two sets of propensity scoring matched cohort. (I)NAT-Surgery vs. Surgery-ACT; (2) nCRT vs. $\mathrm{nCT}$

\begin{tabular}{|c|c|c|c|}
\hline \multirow[t]{2}{*}{ Characteristics } & NAT-Surgery & Surgery-ACT & $P$ value \\
\hline & $\mathrm{n}=389(\%)$ & $\mathrm{n}=389(\%)$ & \\
\hline \multicolumn{4}{|l|}{ Age } \\
\hline mean $( \pm S D)$ & $56.13( \pm 10.42)$ & $55.94( \pm 10.34)$ & 0.992 \\
\hline \multicolumn{4}{|l|}{ Gender } \\
\hline Female & $91(23.4)$ & $107(27.5)$ & \\
\hline Male & $298(76.6)$ & $282(72.5)$ & 0.181 \\
\hline \multicolumn{4}{|l|}{ Tumor location } \\
\hline Upper stomach & $129(33.2)$ & $89(22.9)$ & \\
\hline Lower stomach & $215(55.3)$ & $279(71.7)$ & \\
\hline Whole stomach & $45(11.6)$ & $21(5.4)$ & $<0.001$ \\
\hline \multicolumn{4}{|l|}{ Grade } \\
\hline Well differentiated & $8(2.1)$ & $5(1.3)$ & \\
\hline Moderately differentiated & $90(23.1)$ & $116(29.8)$ & \\
\hline Poor differentiated & $291(74.8)$ & $268(68.9)$ & 0.068 \\
\hline \multicolumn{4}{|l|}{ Clinical T Stage* } \\
\hline $\mathrm{T} 2$ & $24(6.2)$ & $20(5.1)$ & \\
\hline T3 & $121(31.1)$ & $92(23.7)$ & \\
\hline $\mathrm{T} 4$ & $244(62.7)$ & $277(71.2)$ & 0.037 \\
\hline \multicolumn{4}{|l|}{ Clinical N stage* } \\
\hline cN0 & $37(9.5)$ & 45 (11.6) & \\
\hline cN-positive & $352(90.5)$ & $344(88.4)$ & 0.344 \\
\hline \multicolumn{4}{|l|}{ CTNM Stage* } \\
\hline II & $61(15.7)$ & $71(18.3)$ & \\
\hline III & $328(84.3)$ & $318(81.7)$ & 0.331 \\
\hline \multirow[t]{2}{*}{ Characteristics } & $n C R T$ & $n C T$ & $P$ value \\
\hline & $n=100(\%)$ & $n=100(\%)$ & \\
\hline \multicolumn{4}{|l|}{ Age } \\
\hline mean $( \pm \mathrm{SD})$ & $56( \pm 9.2)$ & $54( \pm 11.6)$ & 0.094 \\
\hline \multicolumn{4}{|l|}{ Gender } \\
\hline Female & $16(16.0)$ & $26(26.0)$ & 0.083 \\
\hline Male & $84(84.0)$ & $74(74.0)$ & \\
\hline \multicolumn{4}{|l|}{ Tumor location } \\
\hline Upper stomach & $59(59.0)$ & $59(59.0)$ & 0.5 \\
\hline Lower stomach & $36(36.0)$ & $36(36.0)$ & \\
\hline Whole stomach & $5(5.0)$ & $5(5.0)$ & \\
\hline \multicolumn{4}{|l|}{ Grade } \\
\hline Well differentiated & $4(4.0)$ & $2(2.0)$ & 0.488 \\
\hline Moderately differentiated & $17(17.0)$ & $13(13.0)$ & \\
\hline Poor differentiated & $79(79.0)$ & $85(85.0)$ & \\
\hline \multicolumn{4}{|l|}{ Clinical T stage ${ }^{*}$} \\
\hline $\mathrm{T} 2$ & $1(1.0)$ & $1(1.0)$ & 0.539 \\
\hline T3 & $24(24.0)$ & $31(31.0)$ & \\
\hline T4a & $75(75.0)$ & $68(68.0)$ & \\
\hline \multicolumn{4}{|l|}{ Clinical N stage* } \\
\hline cN0 & $1(1.0)$ & $6(6.0)$ & 0.124 \\
\hline cN-positive & $99(99.0)$ & $94(94.0)$ & \\
\hline \multicolumn{4}{|l|}{ Clinical TNM Stage* } \\
\hline II & $10(10.0)$ & $7(7.0)$ & 0.447 \\
\hline III & $90(90.0)$ & $93(93.0)$ & \\
\hline
\end{tabular}

\section{Pathological assessment}

The pathological outcomes were shown in Table 4. According to the Mandard TRG, the pathological response rate (TRG1-3) was $86.0 \%$ in nCRT group versus $49.0 \%$ in $\mathrm{nCT}$ group $(P<0.001)$. pCR was significantly increased in nCRT group than $\mathrm{nCT}$ group $(17.0 \%$ vs. $4.0 \%, P<0.001)$. Moreover, in nCRT group, significantly lower ypT stage $(P=0.002)$, ypN stage $(P<0.001)$ and lower ypTNM stage $(P<0.001)$ were observed than in $\mathrm{nCT}$ group. The $\mathrm{R} 0$ resection rate and the number of resected lymph nodes were comparable in the two groups.

Table 2. Severe adverse events between two neoadjuvant treatment groups after propensity score matching

\begin{tabular}{llll}
\hline Characteristic & $\begin{array}{l}\mathrm{nCRT}, \\
\mathrm{n}=100(\%)\end{array}$ & $\begin{array}{l}\mathrm{nCT}, \\
\mathrm{n}=100(\%)\end{array}$ & $P$ value \\
\hline \multicolumn{4}{l}{ Grade 3 or 4 adverse events during neoadjuvant treatment } \\
Nausea and vomiting & $7(7.0)$ & $6(6.0)$ & 0.774 \\
Gastrointestinal symptoms & $7(7.0)$ & $7(7.0)$ & 1.000 \\
Neutropaenia/ Thrombocytopaenia & $29(29.0)$ & $14(14.0)$ & $\mathbf{0 . 0 1 0}$ \\
Anemia & $1(1.0)$ & $1(1.0)$ & 1.000 \\
Dyspepsia & $4(4.0)$ & $3(3.0)$ & 1.000 \\
Infection & $0(0.0)$ & $2(2.0)$ & 0.477 \\
Gastrointestinal bleeding & $0(0.0)$ & $1(1.0)$ & 0.238 \\
Hand-foot syndrome & $0(0.0)$ & $0(0.0)$ & 1.000 \\
Gastritis or Esophagitis & $4(4.0)$ & $0(0.0)$ & 0.130 \\
Total number of SAEs & $52(52.0)$ & $34(34.0)$ & $\mathbf{0 . 0 1 0}$ \\
\hline nCRT, neoadjuvant chemoradiation; $\mathrm{nCT}$, neoadjuvant chemotherapy; SAE, severe \\
adverse events.
\end{tabular}

Table 3. Surgical outcomes and postoperative complications of two neoadjuvant groups after propensity score matching

\begin{tabular}{|c|c|c|c|}
\hline Characteristic & nCRT n=100 (\%) & $\mathrm{nCT} n=100(\%)$ & $P$ value \\
\hline \multicolumn{4}{|c|}{ Estimated blood loss, ml } \\
\hline mean $( \pm \mathrm{SD})$ & $170( \pm 157.8)$ & $278( \pm 218.2)$ & $<0.001$ \\
\hline \multicolumn{4}{|c|}{ Surgical time, minutes } \\
\hline mean $( \pm \mathrm{SD})$ & $200( \pm 51.7)$ & $205( \pm 60.8)$ & 0.497 \\
\hline \multicolumn{4}{|c|}{ Intraoperative blood transfusion } \\
\hline & $15(15.0)$ & $29(29.0)$ & 0.017 \\
\hline \multicolumn{4}{|c|}{ Length of postoperative hospital stay } \\
\hline mean $( \pm \mathrm{SD})$ & $11( \pm 3.6)$ & $12( \pm 3.8)$ & 0.005 \\
\hline \multicolumn{4}{|l|}{ Medical expenditure } \\
\hline \multicolumn{4}{|c|}{ Postoperative complications } \\
\hline 30-day mortality & $0(0.0)$ & $1(1.0)$ & 0.238 \\
\hline Abdominal infection & $2(2.0)$ & $4(4.0)$ & 0.678 \\
\hline Anatommotic leakage & $2(2.0)$ & $2(2.0)$ & 1.000 \\
\hline Anatommotic stenosis & $2(2.0)$ & $0(0.0)$ & 0.477 \\
\hline Wound infection & $2(2.0)$ & $4(4.0)$ & 0.678 \\
\hline Abdominal bleeding & $2(2.0)$ & $2(2.0)$ & 1.000 \\
\hline Pneumonia & $1(1.0)$ & $1(1.0)$ & 1.000 \\
\hline $\begin{array}{l}\text { Cardiovascular } \\
\text { complications }\end{array}$ & $0(0.0)$ & $1(1.0)$ & 0.238 \\
\hline $\begin{array}{l}\text { Postoperative } \\
\text { gastrointestinal } \\
\text { dysfunction }\end{array}$ & $1(1.0)$ & $1(1.0)$ & 1.000 \\
\hline Rental failure & $0(0.0)$ & $0(0.0)$ & 1.000 \\
\hline $\begin{array}{l}\text { Total number of } \\
\text { postoperative } \\
\text { complications }\end{array}$ & $9(9.0)$ & $16(16.0)$ & 0.134 \\
\hline
\end{tabular}

\section{Subgroup Survival analysis of nCRT vs. $n C T$}

After a median follow-up of 39.2 months (range, 2.2-131.8 months), median OS in nCRT group and nCT group was 65.5 and 48.2 months, respectively. There was no difference in OS between these two groups (HR, 0.45; 95\% CI, 0.51- 1.11; $P=0.15$; Figure 3A). Patients in nCRT group showed an improvement in DFS (HR, 0.63; 95\% CI, 0.43-0.92; $P=0.014$; Figure 
3B) and LRFS (HR, 0.40; 95\% CI, 0.23-0.69; $P=0.0019$;

Figure $3 \mathrm{C}$ ) than patients in $\mathrm{nCT}$ group.

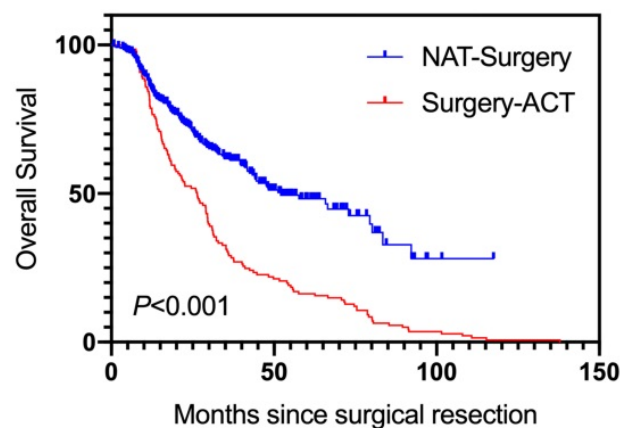

Figure 2. OS by treatment group in LAGC patients. NAT, neoadjuvant therapies; $\mathrm{ACT}$, adjuvant chemotherapy.

\section{A}

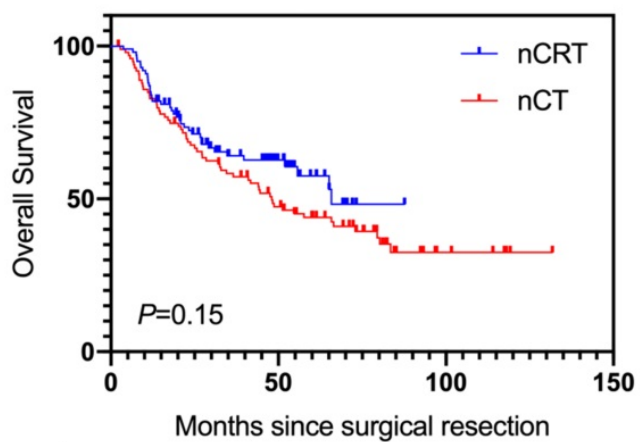

B
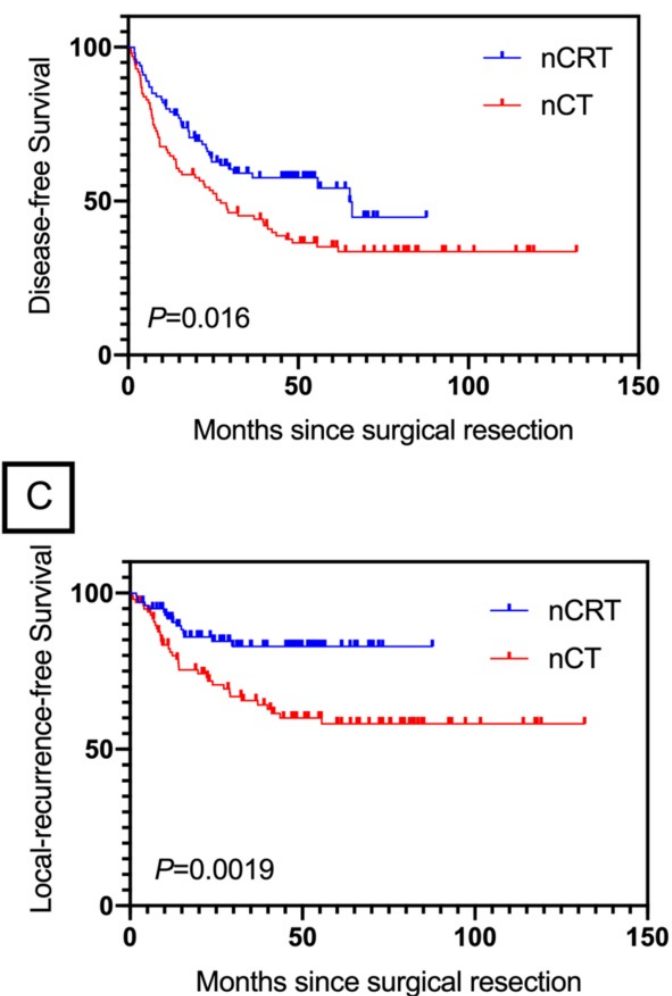

Figure 3. OS (A), DFS (B) and LRFS (C) by treatment group in LAGC patients underwent NAT. nCRT, neoadjuvant chemoradiation; $\mathrm{nCT}$, neoadjuvant chemotherapy.
Table 4. Pathological outcomes of neoadjuvant groups after propensity score matching

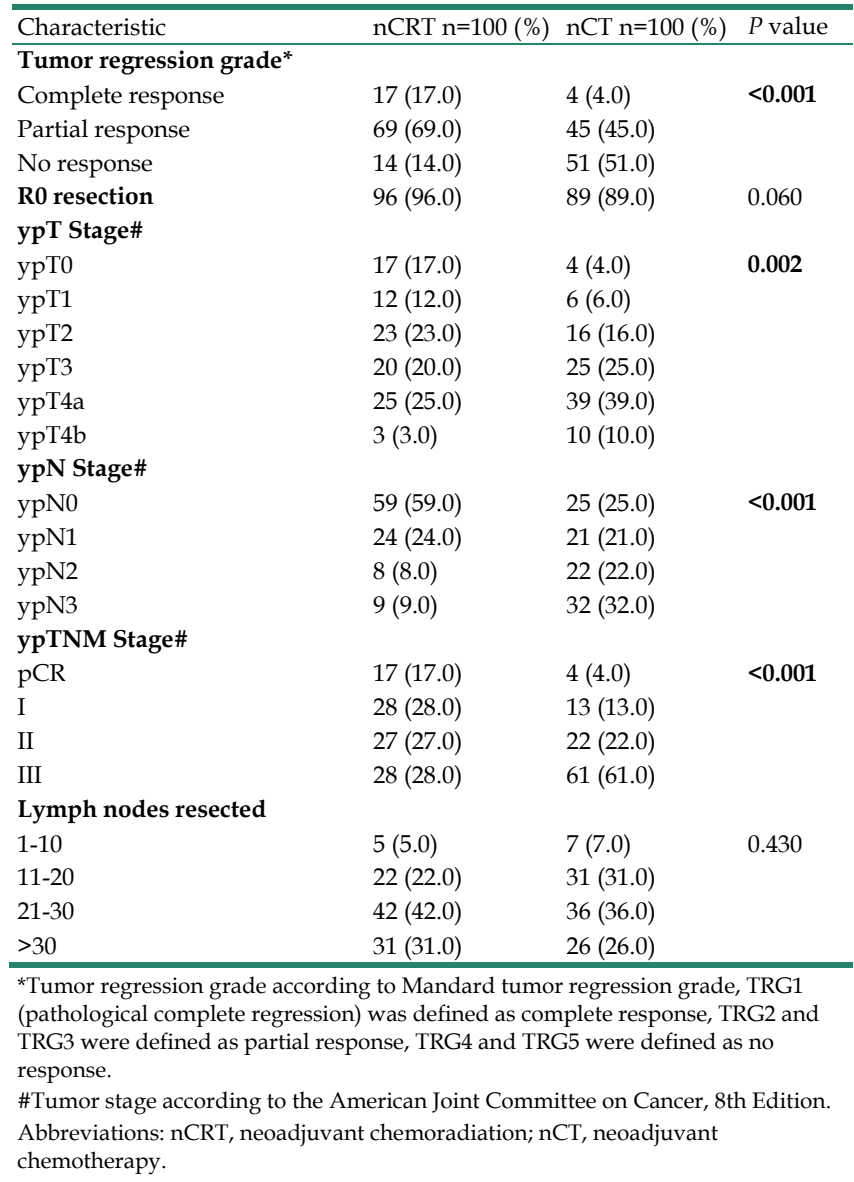

\section{Discussion}

Comparing with surgery alone, survival benefits of neoadjuvant or adjuvant treatments combined with radical surgery for LAGC were shown by several RCTs $[3,6-9,13]$. However, evidence from head to head trails for comparing these two therapeutic concepts was limited or unavailable. The present study had shown a survival advantage of NAT-Surgery compared to Surgery-ACT. The results from our study support the use of neoadjuvant treatments instead of undergoing radical surgery in the first place for LAGC patients. The possible explanation was that preoperative treatments could downstage the tumor, improve pathological responses, eradicate microscopic disease, reduced the risk of local and distant relapses, thus improving the OS [2].

Preoperative chemoradiation has now become the standard of care for patients with oesophageal and gastroesophageal junction tumors, thereby lending further support to its use in gastric cancer [14]. However, preoperative chemoradiotherapy in LAGC patients is still considered investigational. According to our results, the addition of radiotherapy to 
neoadjuvant chemotherapy significantly increased the pathological response rate $(86.0 \%$ vs. $49.0 \%, P<0.001)$ and $\mathrm{pCR}$ rate $(17.0 \%$ vs. $4.0 \%, P<0.001)$. These data consisted with the previous results from several studies suggested a superior regression grade rate after preoperative chemoradiation in comparison with that seen with chemotherapy alone [15-17]. Moreover, nCRT was also correlated with a lower ypT, ypN and ypTNM stage then nCT group in the current study. This suggests that chemoradiation therapy induced a higher response rate not only in the tumor wall but also in the lymph nodes, which is also consistent with conclusion of a previous study that preoperative chemoradiation increases the likelihood of achieving favorable histopathological features [16].

In the present study, we found that nCRT achieved significantly better DFS $(P=0.014)$ and LRFS $(P=0.0019)$ than nCT. These results support the current hypothesis that adding chemoradiation to standard perioperative chemotherapy will achieve even greater survival gains in LAGC patients. To our knowledge, the improvement for local disease control and distant recurrence after adding radiation to neoadjuvant therapy in LAGC patients has not been described previously. A possible reason for this difference could be that radiation eradicates the microscopic disease and malignant cells in the irradiated volume are radiosensitised and microscopic deposits outside are treated by adding chemotherapy [5].

The OS benefits of the use of nCRT in LAGC patients' remain controversial. Several studies have shown that the addition of radiation to neoadjuvant chemotherapy does not affect the OS [17-19]. On the other hand, several researches reported a survival advantage for preoperative chemotherapy compared with preoperative chemotherapy for adenocarcinoma of EGJ [20, 21]. Although, in the current study, there was no difference in OS between nCRT and nCT group, the analysis of survival revealed a type revealed a trend towards improved survival after the addition of radiotherapy among LAGC patients. This fact may be partly attributable to the relatively short follow-up period for the group of nCRT.

One of the major arguments with the use of nCRT is toxicity. Although, in the present study, the rate of grade 3 or 4 adverse events was significantly higher in nCRT group $(52.0 \%$ vs. $34.0 \%, P=0.010)$, no death event during neoadjuvant treatment was recorded in both $\mathrm{nCRT}$ and $\mathrm{nCT}$ group. It was believed that nCRT could increase the difficulty of surgery and postoperative complications [21, 22]. Interestingly, the current study revealed a less mean blood loss, lower the intraoperative blood transfusion rate and shorter mean postoperative hospital stay in
nCRT group. Moreover, the postoperative complications in both groups were comparable. Nevertheless, these data should be viewed with caution, given its retrospective exploratory nature and the limited number of patients analyzed.

Strengths and limitations should be considered when interpreting the study results. The present study was a retrospective cohort study using PSM method, which minimized the potential selection bias and confounding. Our study, for the first time, reported that nCRT had survival benefits of DFS and LRFS compared with nCT among LAGC patients. However, some patients were missing crucial clinical data that may have also affected survival outcomes such as comorbidities. Moreover, our follow-up was relatively short. Finally, this was a single-institutional analysis with significant treatment heterogeneity.

\section{Conclusion}

In conclusion, NAT improved survival outcomes among LAGC patients over surgery followed by adjuvant chemotherapy. In comparison with $\mathrm{nCT}$, nCRT resulted in higher pCR rate, better DFS and LRFS, without significantly affecting OS.

\section{Supplementary Material}

Supplementary table S1.

http://www.jcancer.org/v12p0379s1.pdf

\section{Acknowledgements}

\section{Funding}

This study received funding from Beijing Hope Run Special Fund of Cancer Foundation of China (No. LC2017L01), the Special Research Projects for Capital Health Development (No. 2014-1-2151) and National Key R\&D Program of China (No. 2017YFC0908300).

\section{Human rights statement and informed consent}

All procedures followed were in accordance with the ethical standards of the responsible committee on human experimentation (institutional and national) and with the Helsinki Declaration of 1964 and later versions. Informed consent to be included in the study, or the equivalent, was obtained from all patients.

\section{Contributions}

Conception and design: TB Wang, YT Chen, LL Zhao, AP Zhou, J Jin, DB Zhao; Collection and assembly of data: TB Wang, LL Zhao, H Zhou, CR $\mathrm{Wu}$; Data analysis and interpretation: TB Wang, YT Chen, LL Zhao; Manuscript writing: All authors; Final approval of the manuscript: All authors. 


\section{Competing Interests}

The authors have declared that no competing interest exists.

\section{References}

1. Bray F, Ferlay J, Soerjomataram I, Siegel RL, Torre LA, Jemal A. Global cancer statistics 2018: GLOBOCAN estimates of incidence and mortality worldwide for 36 cancers in 185 countries. CA Cancer J Clin. 2018; 68: 394-424.

2. Petrillo A, Pompella L, Tirino G, Pappalardo A, Laterza MM, Caterino M, et al. Perioperative Treatment in Resectable Gastric Cancer: Current Perspectives and Future Directions. Cancers (Basel). 2019; 11.

3. Ychou M, Boige $V$, Pignon JP, Conroy $T$, Bouche $O$, Lebreton $G$, et al Perioperative chemotherapy compared with surgery alone for resectable gastroesophageal adenocarcinoma: an FNCLCC and FFCD multicenter phase III trial. J Clin Oncol. 2011; 29: 1715-21.

4. Nashimoto A, Akazawa K, Isobe Y, Miyashiro I, Katai H, Kodera Y, et al Gastric cancer treated in 2002 in Japan: 2009 annual report of the JGCA nationwide registry. Gastric Cancer. 2013; 16: 1-27.

5. Van Cutsem E, Sagaert X, Topal B, Haustermans K, Prenen H. Gastric cancer. Lancet. 2016; 388: 2654-64.

6. Macdonald, John, S., Smalley, Stephen, R., et al. Chemoradiotherapy after Surgery Compared with Surgery Alone for Adenocarcinoma of the Stomach or Gastroesophageal Junction. New England Journal of Medicine. 2001.

7. Cunningham, David, Allum, William H, Stenning, Sally P, et al. Perioperative Chemotherapy versus Surgery Alone for Resectable Gastroesophageal Cancer. New England Journal of Medicine. 2006.

8. Sasako M, Sakuramoto S, Katai H, Kinoshita T, Furukawa H, Yamaguchi T, et al. Five-year outcomes of a randomized phase III trial comparing adjuvant chemotherapy with S-1 versus surgery alone in stage II or III gastric cancer. Journal of Clinical Oncology Official Journal of the American Society of Clinical Oncology. 2011; 29: 4387.

9. Bang YJ, Kim YW, Yang HK, Chung HC, Park YK, Lee KH, et al. Adjuvant capecitabine and oxaliplatin for gastric cancer after D2 gastrectomy (CLASSIC): a phase 3 open-label, randomised controlled trial. Lancet. 2012; 379: 315-21.

10. Watanabe H, Okada M, Kaji Y, Satouchi M, Sato Y, Yamabe Y, et al. New response evaluation criteria in solid tumours-revised RECIST guideline (version 1.1). Gan To Kagaku Ryoho. 2009; 36: 2495-501.

11. Clavien PA, Barkun J, de Oliveira ML, Vauthey JN, Dindo D, Schulick RD, et al. The Clavien-Dindo classification of surgical complications: five-year experience. Ann Surg. 2009; 250: 187-96.

12. Mandard AM, Dalibard F, Mandard JC, Marnay J, Henry-Amar M, Petiot JF, et al. Pathologic assessment of tumor regression after preoperative chemoradiotherapy of esophageal carcinoma. Clinicopathologic correlations. Cancer. 1994; 73: 2680-6.

13. Lee J, Lim DH, Kim S, Park SH, Park JO, Park YS, et al Phase III trial comparing capecitabine plus cisplatin versus capecitabine plus cisplatin with concurrent capecitabine radiotherapy in completely resected gastric cancer with D2 lymph node dissection: the ARTIST trial. J Clin Oncol. 2012; 30: 268-73.

14. van Hagen $\mathrm{P}$, Hulshof MC, van Lanschot JJ, Steyerberg EW, van Berge Henegouwen MI, Wijnhoven BP, et al. Preoperative chemoradiotherapy for esophageal or junctional cancer. N Engl J Med. 2012; 366: 2074-84.

15. Ikoma N, Das P, Hofstetter W, Ajani JA, Estrella JS, Chen HC, et al Preoperative chemoradiation therapy induces primary-tumor complete response more frequently than chemotherapy alone in gastric cancer: analyses of the National Cancer Database 2006-2014 using propensity score matching. Gastric Cancer. 2018; 21: 1004-13.

16. Martin-Romano P, Sola JJ, Diaz-Gonzalez JA, Chopitea A, Iragorri Y, Martínez-Regueira F, et al. Role of histological regression grade after two neoadjuvant approaches with or without radiotherapy in locally advanced gastric cancer. Br J Cancer. 2016; 115: 655-63.

17. Klevebro $F$, Alexandersson von Döbeln $G$, Wang $N$, Johnsen $G$, Jacobsen $A B$, Friesland $S$, et al. A randomized clinical trial of neoadjuvant chemotherapy versus neoadjuvant chemoradiotherapy for cancer of the oesophagus or gastro-oesophageal junction. Ann Oncol. 2016; 27: 660-7.

18. Burmeister $\mathrm{BH}$, Thomas JM, Burmeister EA, Walpole ET, Harvey JA, Thomson $\mathrm{DB}$, et al. Is concurrent radiation therapy required in patients receiving preoperative chemotherapy for adenocarcinoma of the oesophagus? A randomised phase II trial. Eur J Cancer. 2011; 47: 354-60.

19. Sjoquist KM, Burmeister BH, Smithers BM, Zalcberg JR, Simes RJ, Barbour A, et al. Survival after neoadjuvant chemotherapy or chemoradiotherapy for resectable oesophageal carcinoma: an updated meta-analysis. Lancet Oncol. 2011; 12: 681-92.

20. Stahl M, Walz MK, Stuschke M, Lehmann N, Meyer HJ, Riera-Knorrenschild J, et al. Phase III comparison of preoperative chemotherapy compared with chemoradiotherapy in patients with locally advanced adenocarcinoma of the esophagogastric junction. J Clin Oncol. 2009; 27: 851-6.

21. Stahl M, Walz MK, Riera-Knorrenschild J, Stuschke M, Sandermann A, Bitzer $\mathrm{M}$, et al. Preoperative chemotherapy versus chemoradiotherapy in locally advanced adenocarcinomas of the oesophagogastric junction (POET):
Long-term results of a controlled randomised trial. Eur J Cancer. 2017; 81: 183-90.

22. Klevebro F, Johnsen G, Johnson E, Viste A, Myrnäs T, Szabo E, et al. Morbidity and mortality after surgery for cancer of the oesophagus and gastro-oesophageal junction: A randomized clinical trial of neoadjuvant chemotherapy vs. neoadjuvant chemoradiation. Eur J Surg Oncol. 2015; 41: $920-6$ 